\title{
Increased myo-inositol in the posterior cingulate cortex in first-episode major depressive patients
}

\author{
Lily Magally Granados-Domínguez ${ }^{1,2}$, Enrique O. Flores-Gutiérrez², Sarael Alcauter², \\ Juan José Cervantes ${ }^{2}$, Marina Torres-Álvarez ${ }^{2}$, María Corsi-Cabrera ${ }^{*}$ \\ ${ }^{1}$ Laboratory for Sleep Research, Faculty of Psychology, Universidad Nacional Autónoma de México (UNAM), Mexico City, Mexico \\ ${ }^{2}$ Sub-Department of Clinical Investigations, Instituto Nacional de Psiquiatría Ramón de la Fuente Muñíz, Mexico City, Mexico \\ Email: corsi@unam.mx
}

Received 27 March 2013; revised 10 May 2013; accepted 23 May 2013

Copyright (C) 2013 Lily Magally Granados-Domínguez et al. This is an open access article distributed under the Creative Commons Attribution License, which permits unrestricted use, distribution, and reproduction in any medium, provided the original work is properly cited.

\begin{abstract}
Major depressive disorder (MDD) is a severe, disabling pathology characterized, in addition to affective, cognitive and motor symptoms, by self-focused attention and rumination. During recursive self-focused processes and rumination, the posterior cingulate cortex (PCC) is activated. In vivo proton magnetic resonance spectroscopy (MRS) is a noninvasive imaging technique that can directly assess living biochemistry in localized brain regions. The aim of this study, therefore, was to use ${ }^{1} \mathrm{H}$-MRS as a means of analyzing brain metabolites in the PCC of a group of first-episode, unmedicated MDD patients. PCC metabolite levels were analyzed at 3-T in a single voxel located bilaterally over the PCC in 7 patients diagnosed for the first time with MDD and with no previous pharmacological treatment, as well as in 9 control subjects. Differences in metabolite levels between groups were compared using independent t-tests. Myo-inositol was significantly higher, and NAA + NAAG/Cr significantly lower, in MDD patients than in controls. The other brain metabolites showed no statistical differences. The present results suggest that alterations in PCC metabolite levels are likely involved in MDD pathophysiology, and may help to improve our understanding of MDD and the role of the PCC in some symptoms of depression.
\end{abstract}

Keywords: Major Depressive Disorder; Posterior Cingulate Cortex; Metabolites; ${ }^{1}$ H-MRS; Myo-Inositol; N-Acetyl-Aspartate

\section{INTRODUCTION}

Major depressive disorder (MDD) is a complex pathol- ogy with cognitive and emotional symptoms; is considered one of the most prevalent and disabling of all psychiatric disorders [1]. In addition to affective symptoms such as low mood, anhedonia, poor motivation, impaired psychomotor activity and reduced energy, MDD is characterized by self-focused attention [2] and rumination; i.e., recurrent obsessive thoughts on one's negative mood, guilt, death, failure or inadequacy [3], and on the possible causes and consequences of these symptoms [4]. Converging evidence from clinical, neuropathological and neuroimaging studies of depression have shown structural, metabolic and electroencephalographic alterations in specific brain regions linked to distributed networks involved in cognitive and emotional modulation. MDD has been associated with decreased activity in prefrontal areas, including the dorsolateral and ventrolateral prefrontal cortices, the parietal lobule and the dorsal anterior and posterior cingulate cortices, and with increased activation of limbic and paralimbic areas, such as the anterior subgenual cingulate, the insula, the hippocampus, the hypothalamus and the amygdala [5-7]. Some of these regions overlap two brain networks: the default-mode network [8] and the cortical midline network, both of which are involved in self-referential processes and depression [2,9]. Activation of the default-mode network has been associated with introspective states, such as processes implying reference to oneself [10], episodic memory and rumination [11], while some structures of the midline network are activated in self-related tasks [12]. Brain regions participating in the default-mode network have been found to be less deactivated in depressed patients at rest $[13,14]$, a decreased deactivation that has been correlated with the severity of depression and feelings of hopelessness [15]. In recent years, the posterior cingulate cortex (PCC) has become a focus of 
attention for two reasons: first, it has been linked to self-focus and rumination; and, second, it forms part of both of these networks. The PCC has been found to be activated in healthy subjects during information retrieval from episodic [16] and autobiographic memories [17], as well as during self-referential processing $[10,18,19]$, and rumination [20]. Interestingly, some neuroimaging studies have found both structural and activity alterations in the PCC of MDD patients; for example, MDD patients have reduced cortical thickness [21], decreased gray matter volume [22] and lesser cortical folding and gyrification index over PCC [23]. MDD patients show electroencephalographic hypoactivation over the posterior cingulate while at rest [24], and decreased blood flow with sad, compared to neutral word retrieval [25]; whereas they present greater activation in the PCC than controls during rumination compared to the abstract distraction condition [20], and to sad faces compared to healthy volunteers before antidepressant treatment. This highly-activated state of the PCC decreases after effective antidepressive pharmacological treatment $[5,26]$. In addition to numerous abnormalities in brain morphology and neurotransmitter systems, a growing body of evidence suggests that metabolite levels in several brain regions, as assessed by in vivo proton magnetic resonance spectroscopy (MRS), are altered in MDD and may play an important role in the pathophysiology of depression. Magnetic resonance spectroscopy is a noninvasive imaging technique that can directly assess the most abundant metabolites in localized brain regions [27]. Changes in metabolite levels can be considered markers of the functional integrity and viability of brain tissue, and have been used successfully in the study of psychiatric disorders [28]. Most of the MRS studies in cases of depression have focused on anterior brain areas. Myoinositol (mI), one of the metabolites analyzed by MRS, is a precursor molecule for several brain metabolites. It participates in signaling processes in the phosphatidylcholine system and is involved in the regulation of cellular osmolarity; therefore, it has been considered as a possible marker of inflammatory responses in the brain and of glial cell loss [27,29]. Levels of $\mathrm{mI}$ have been found to be lower in the anterior cingulate cortex [21], as has the $\mathrm{mI} / \mathrm{Cr}$ (creatine) ratio in the left and right prefrontal regions [30] and in the prefrontal and anterior cingulate cortices in medicated patients after several weeks of wash-out [31]. In contrast, higher levels have been seen in the medial prefrontal cortex and pregenual cingulate cortex of medication-free, fully-recovered patients [32] and in the left dorsolateral prefrontal cortex in treatment-naïve, first-episode, female patients and after antidepressant treatment [33]. N-acetylaspartate (NAA) and choline compounds (Cho) participate in lipid biosynthesis; hence they are considered putative markers of neu- ronal integrity [27]. First-episode and treatment-naïve patients with MDD showed significantly lower NAA/Cr ratios in the DLPFC compared to control subjects [34] and to recurrently-remitted and chronic patients, while choline compounds show increased values in chronically-depressed patients with prolonged illness duration [35]. However, most of the MRS studies of MDD have reported no differences in NAA [27,31]. Glu and GABA have been reported to be lower in dorsomedial, ventromedial and anterolateral prefrontal regions in nonmedicated MDD patients [36], while the decreased levels of Glu and $\mathrm{Gln} / \mathrm{Cr}$ in the pregenual region of the cingulate cortex are associated with the severity of depressive episodes [37]. However, Glu decreases in both remitted and chronic, non-remitted MDD patients compared to first-episode and control subjects [35]. These apparently discrepant results in MRS studies of depression may be explained by the variability and specific characteristics of the different patient groups studied, including different age, female vs. male, drug-naïve vs. medicated, firstepisode vs. chronic depressives, and remitters vs. nonremitters, as well as to the sensitivity of the methodology utilized to assess metabolite levels. Despite the many studies reporting structural and activation alterations of the PCC and an association of the PCC with rumination and self-focus in MDD, little is yet known about the biochemical composition of the PCC in MDD. The aim of the present exploratory study, therefore, was to use ${ }^{1} \mathrm{H}$-MRS at 3-T to examine metabolite levels in the PCC in first-episode, drug-naïve patients with MDD. We examined the metabolites in a voxel centered on the PCC, hypothesizing that because the PCC presents structural and activation abnormalities, metabolite levels will be altered in MDD patients compared to healthy individuals.

\section{METHODS AND MATERIALS}

\subsection{Subjects}

Eight adult male patients from 21 to 46 years of age participated in this ${ }^{1} \mathrm{H}-\mathrm{MRS}$ study. To avoid possible confounding factors derived from plastic changes in brain metabolites due to long-term illness evolution, or those introduced by lifelong pharmacological use, only firstepisode, unipolar-diagnosed MDD (MDDG) and naïvetreatment patients were included. Patients were recruited from the Clinical Service of Instituto Nacional de Psiquiatría Ramón de la Fuente Muñiz (INPRFM) in Mexico City. A psychiatrist confirmed both the primary diagnosis of unipolar MDD and the absence of any other Axis I psychiatric disorders, according to DSM-IV criteria [38]. A control group $(C G)(n=9)$ within the same age range (23 to 47 years old) was recruited through announcements posted around the INPRFM. Healthy controls were free of medication and had no current symp- 
toms or medical histories of psychiatric, neurological or medical disorders. They were screened through a diagnostic interview and were subjected to the same tests as the MDD patients. To confirm the absence of psychiatric disorders in $\mathrm{CG}$ and comorbidity in the MDDG, all participants were screened using the SCL-90R questionnaire, which gives a global severity index derived from nine subscales that explore somatization, obsessivecompulsive symptoms, interpersonal sensitivity, depression, anxiety, hostility, phobic anxiety, paranoia, ideation, and psychoticism [39]. The Hamilton Depression Scale [40] and Beck Depression Inventory [41] were used to confirm diagnoses and determine the severity of MDD, as well as its absence in CG. Only patients with moderate-to-severe depression according to the Hamilton Depression Scale (score > 14) and the Beck Depression Inventory (score $>10$ ) were included. Control subjects had to be within normal range on both scales (i.e., Beck $<7$; Hamilton $<10$ ). None of the participants in CG met the criteria for either current or past MDD episodes. The exclusion criteria for MDD patients included: 1) other Axis I psychiatric disorders and symptoms; 2) a history of alcohol or substance abuse within the 6 months prior to entering the study; 3 ) the presence of neurological disorders and physical illness. The exclusion criteria for healthy controls included psychiatric illness in first-degree relatives, and current or past medical or neurological illness. Exclusion criteria for all potential participants were counter-indications for ${ }^{1} \mathrm{H}$-MRS. All participants gave their informed, written consent after a full explanation of the study protocol, which was approved previously by the Ethics Committee of the INPRFM.

\subsection{Procedure}

Once the Clinical Service of the INPRFM had screened the MDD patients to determine those who satisfied the inclusion and exclusion criteria, those individuals were scheduled for a single session at the Cerebral Imaging Area of the INPRFM just prior to beginning antidepressant treatment. Evaluations and scanning were done in a single session that was divided into two phases. In the first, subjects were assessed by a trained psychiatrist (J.J.C.) in accordance with the Hamilton Rating Scale for Depression, the Beck Depression Inventory, and the SCL-90R questionnaire. In the second phase, the actual ${ }^{1} \mathrm{H}-\mathrm{MRS}$ scanning was conducted. Control subjects followed exactly the same procedure.

\section{3. ${ }^{1}$ H-MRS Scanning}

Localized ${ }^{1} \mathrm{H}$-MRS was performed in a routine clinical MR scanner Achieva Quasar Dual MRI System (Phillips Medical Systems, Eindhove, Holland) at 3.0 Tesla. Each subject participated in a single voxel spectroscopy scan located in the posterior cingulate cortex (PCC). As an anatomical reference of high resolution and contrast, it was included the T1 image adquisition with inverse recovery pulses (IR), (repetition time) $\mathrm{TR}=2949 \mathrm{~ms}$, (echo time) $\mathrm{TE}=15 \mathrm{~ms}$, inversion time (IT) $=400 \mathrm{~ms}$. A $20 \times 20 \times 20 \mathrm{~mm}^{3}$ voxel was placed bilaterally on the PCC aligned tangential to the splenium of the corpus callosum in a sagittal plane, centered on the midline (Figure 1). Single-voxel ${ }^{1} \mathrm{H}-\mathrm{MRS}$ spectroscopy was conducted using a short echo PRESS (Point Resolved Spectroscopy Sequence) ( $\mathrm{TE}=40 \mathrm{~ms}, \mathrm{TR}=2000 \mathrm{~ms}$, NS $=64)$. Quantification of metabolic intensities was performed by using the LCModel [7] with water suppress peak as reference [42]. The Linear Combination of Metabolite Basis Spectra (LCModel) normalizes the metabolite spectra obtained using the water suppressed peak as reference [42].

\subsection{Statistical Analysis}

Statistical analysis was performed with commercial SPSS software (SPSS 17.0 for Windows). Student-T tests for independent samples were used to compare statistical differences between MDDG and CG for each of the metabolites assessed in the PCC.

\section{RESULTS}

One MDD patient had to be excluded due to claustrophobia, which caused the scanning sessions to be interrupted; thus 7 of the 8 MDD patients and all of the control subjects completed the study. The results obtained from the Hamilton Depression Scale (MDDG: mean = 21.11, $\mathrm{SD}=5.32 ; \mathrm{CG}$ : mean $=1.33, \mathrm{SD}=1.52 ; \mathrm{t}=4.40$; $\mathrm{p}<0.0009)$ and the Beck Depression Inventory (MDDG: mean $=27.44, \mathrm{SD}=6.54 ; \mathrm{CG}$ : mean $=1.66, \mathrm{SD}=1.52 ; \mathrm{t}$ $=8.42 ; \mathrm{p}<0.00001)$ showed the expected significant differences between MDDG and CG, thus confirming the diagnoses of major depressive disorder in the former and the absence of depression in the latter. Results of the SCL-90R questionnaire confirmed the absence of psychiatric disorders in $\mathrm{CG}$ and of comorbidities in MDDG (MDDG: mean $=1.48, \mathrm{SD}=0.80 ; \mathrm{CG}$ : mean $=0.071$, $\mathrm{SD}=0.02 ; \mathrm{t}=2.36 ; \mathrm{p}=0.16)$. Location of the voxel and Representative spectra and LCModel fits are illustrated in Figure 1. The results of ${ }^{1} \mathrm{H}-\mathrm{MRS}$ scanning for the metabolites assessed are shown in Table $\mathbf{1}$ and Figure 2. Significantly increased levels of $\mathrm{mI}$ and of the $\mathrm{mI} / \mathrm{Cr}$ ratio, together with a decreased NAA + NAAG/Cr ratio in the PCC were observed in the MDD group compared to healthy controls. There were no significant differences between the groups with respect to the direct measurements of $\mathrm{Cr}, \mathrm{Cr}+\mathrm{PCr}$, Glu, Gln, GPC, GPC + PCh and NAA, or in the metabolite-to-Cr ratios of Glu/Cr, GPC + $\mathrm{PCh} / \mathrm{Cr}, \mathrm{NAA} / \mathrm{Cr}$. 


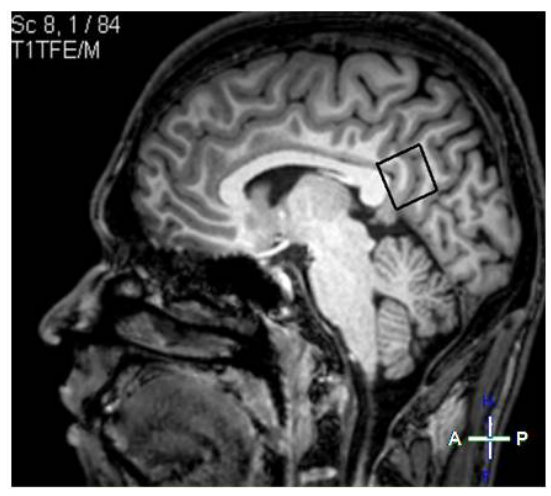

(a)

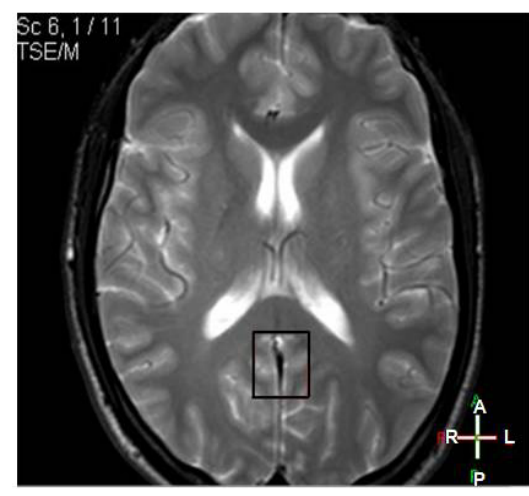

(b)

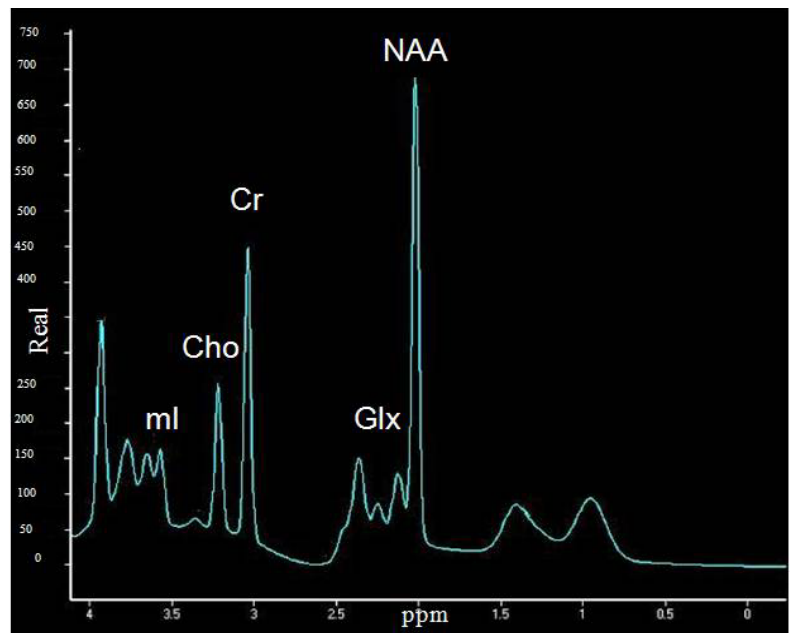

(c)

Figure 1. Schematic of voxel location over bilateral posterior cingulate cortex, sagittal view (a) and axial view (b). Representative spectra of a control subject (c).

Table 1. Absolute metabolite concentrations registered by ${ }^{1} \mathrm{H}-\mathrm{MRS}$ and analyzed by LCModel for Major. Depressive Disorder Group (MDDG) and Control Group (CG).

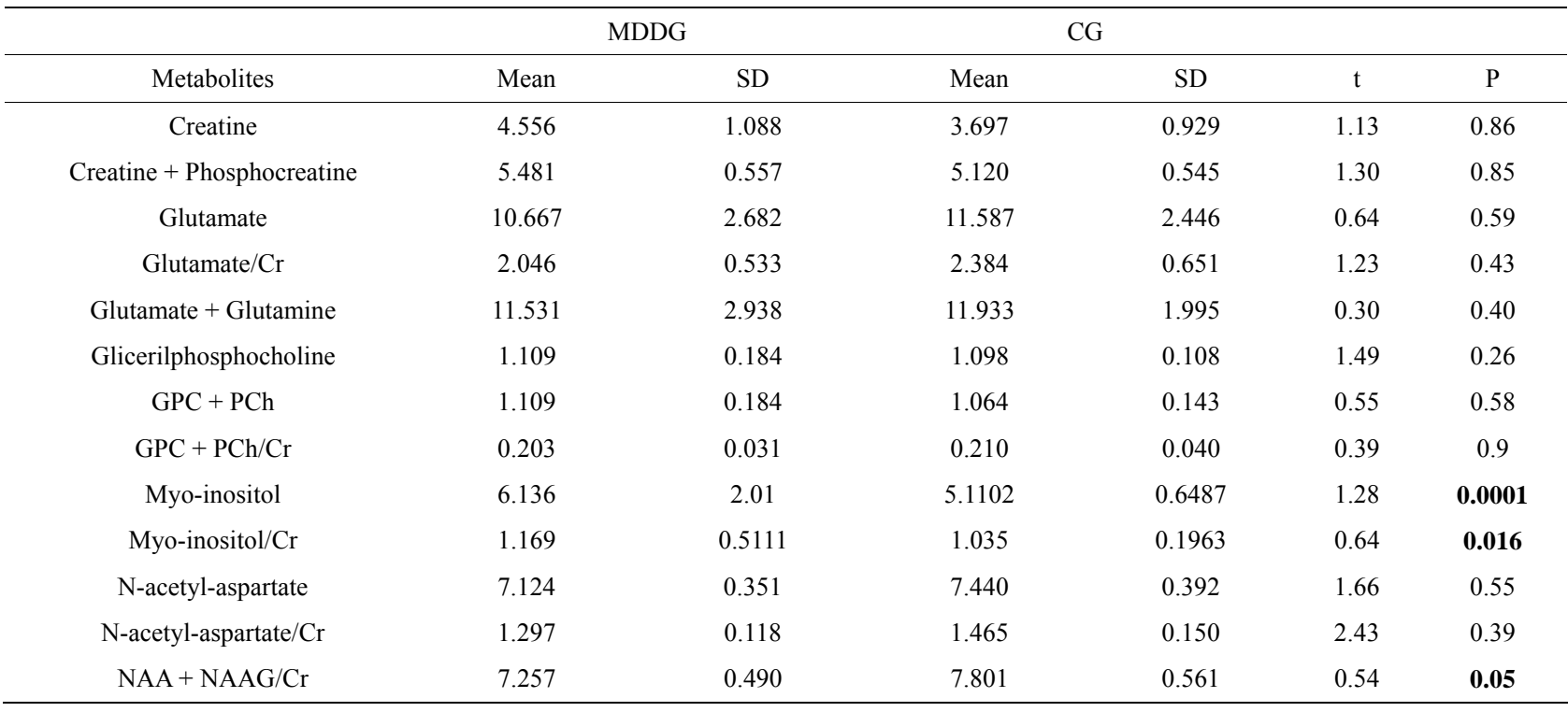

SD: Standard Deviation; t: Student-t test for independent groups. GPC+PCh: glicerylphosphocholine; Cr: creatine; NAAG: N-acetyl-aspartate-glutamate; $\mathrm{p}<$ 0.05 in bold. 


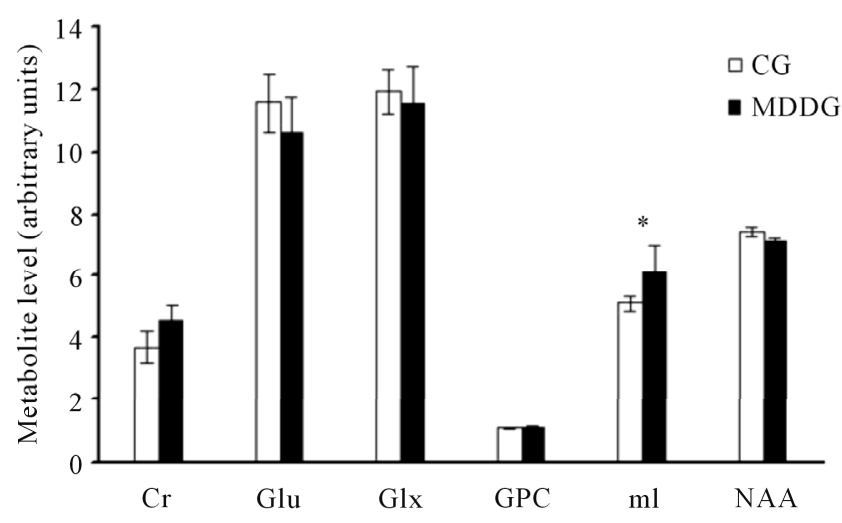

(a)

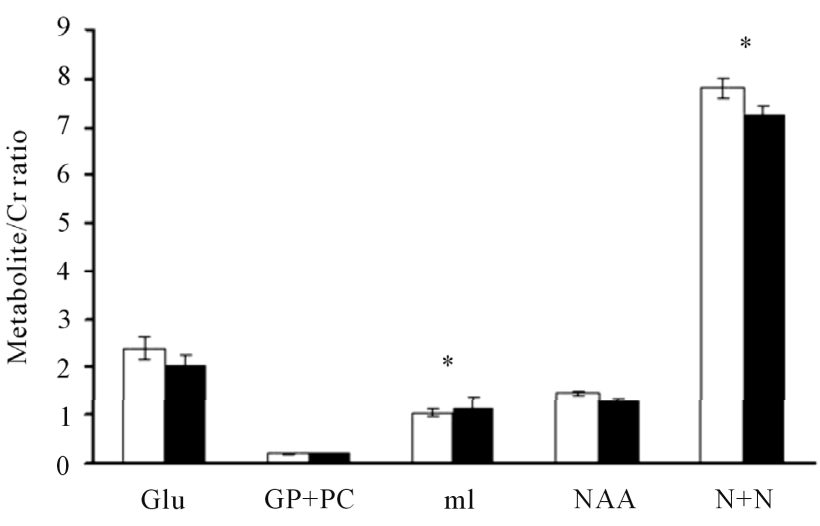

(b)

Figure 2. Mean and standard error of metabolite levels (a) and metabolite to Cr levels (b) for the control group (CG) and the major depressive disorder patients (MDDG). Asterisks above bars indicate significant differences between groups $(\mathrm{p}<0.05)$.

\section{DISCUSSION}

The main finding of this exploratory study of metabolite levels in the PCC in major depression is that the $\mathrm{mI}$ level was significantly higher, while the NAA + NAAG/Cr ratio decreased in the PCC of first-episode, treatmentnaïve male MDD patients; results that suggest the involvement of the PCC in the pathophysiology of the early stages of depression. Both the increased $\mathrm{mI}$ and the decreased NAA + NAAG/Cr levels indicate an alteration in PCC metabolite states that may well be associated with altered brain cell metabolism. Although the decrease observed in NAA+NAAG was only significant to the $\mathrm{Cr}$ ratio, $\mathrm{Cr}$ was not significantly different in MDD patients. NAA has been associated with neuronal integrity [27], whereas increases in $\mathrm{mI}$ content have been interpreted as representing either glial proliferation or an increase in glial cell size and, therefore, have been considered markers of inflammation in the brain [29]. It has also been demonstrated that high levels of $\mathrm{mI}$ are present in some types of neurons, where it is involved in second messenger signaling [29]. Though the present methodology is not capable of differentiating between $\mathrm{mI}$ level in glial or neuronal cells, or of determining whether this increase reflects compensatory changes related to other pathogenic processes (further research is needed), these results do indicate an alteration in PCC metabolite levels and thus support the involvement of the PCC in depression. Rumination and self-focused attention constitute important symptoms of depression and are considered risk factors for the onset and course of depression $[2,43]$. The findings of altered metabolite levels in the PCC in this study support the notion that the PCC may be involved in pathological rumination and self-focused attention in depression; however, metabolite levels in the PCC of depressed patients have not yet been assessed; thus the increase in the resonance peak of $\mathrm{mI}$ in the PCC cannot be directly compared with other studies. Nevertheless, the present results of increased $\mathrm{mI}$ levels in the PCC in MDDG are in line with studies showing morphological alterations [21,23], lower electroencephalographic activation at rest [24], and higher metabolic activation during rumination [20] in MDD patients. The literature contains reports of functional hyper-connectivity in the PCC and the subgenual-cingulate cortex, correlated with behavioral measures of rumination and brooding in relation to the severity of depression [24]; and of the PCC with the dorsomedial prefrontal cortex, correlated with depressive symptoms in MDD [14]. The present results actually suggests an imbalance between the anterior and posterior regions of the midline system that concords with the proposal that altered functional connectivity underlies some aspects of emotional deregulation [14]. Moreover, this agrees with studies postulating that the effects of lithium therapy in mania and depression may be a result of $\mathrm{mI}$ depletion [44]. Most previous studies on brain metabolites have been carried out using 1.5-Tesla scanners, but the present study used high-field imaging at 3.0 Tesla and brain water as a referencing method to provide a substantial increase in sensitivity and a better possibility of assigning resonance peaks directly, and not as a ratio to $\mathrm{Cr}$. This approach makes it possible to determine whether a higher measured level of $\mathrm{mI}$ is due to a lower level of $\mathrm{Cr}$ or to a real increase of $\mathrm{mI}$.

\section{Limitations}

The present data must be considered as preliminary given the small number of patients assessed and, undoubtedly, need to be corroborated in larger groups of major depression patients. Unfortunately, due to the nature of the INPRFM, the majority of patients who seek treatment have already experienced multiple depressive episodes and comorbidity with other Axis I psychiatric disorders [38], and also have long histories of drug use; thus, it was not possible to locate more than these 8 
young, first-episode patients that were free of Axis I comorbidities and had not received any pharmacological treatment during at least an interval of one year. Because of the confounding effects introduced by the diversity of types of depression, illness duration, and histories of antidepressant treatment, we preferred to maintain group homogeneity rather than include a larger number of patients. Despite this shortcoming, and given the lack of studies on metabolite states in PCC in depression, we considered it important to communicate the present findings. Our MDD patients did not differ from healthy controls in the other metabolites measured. The lack of any such significant differences agrees with studies of anterior brain regions and with similar conditions as our patients in the MDDG that found no differences in other brain metabolites like Glu, Cr [31-34,37], NAA [31,33, $34]$ or Cho $[21,31,33]$; however, the possibility that these variations are due to the small number of participants cannot be discarded. In conclusion, to the best of our knowledge this is the first evidence of metabolite alterations in the PCC in moderate-to-severe depression in first-episode, medication-naïve patients. Our results suggest that alterations in PCC metabolite levels are likely involved in MDD pathophysiology and may help improve our understanding of this pathophysiology and the role of PCC in some symptoms of depression.

\section{ACKNOWLEDGEMENTS}

Lily M. Granados-Domínguez received a grant from CONACYT for graduate studies. This work constitutes part of an academic thesis developed for the Ph.D. program in Psychology at the UNAM. Mr. Paul Kersey corrected the English version of the manuscript. We thank Margarita Lopez Titla of the Instituto Nacional de Psiquiatría Ramón de la Fuente Muñiz for the ${ }^{1} \mathrm{H}-\mathrm{MRS}$ images and Ph.D. Irma Yolanda del Río-Portilla for her valuable support.

\section{REFERENCES}

[1] World Health Organization (2001) The world health report.

[2] Northoff, G. (2007) Psychopathology and pathophysiology of the self in depression-Neuropsychiatric hypothesis. Journal of Affective Disorders, 104, 1-14. doi:10.1016/j.jad.2007.02.012

[3] Drevets, W.C., Videen, T.O., Price, J.L., Preskorn, S.H., Carmichael, S.T. and Raichle, M.E. (1992) A functional anatomical study of unipolar depression. Journal of Neuroscience, 12, 3628-3641.

[4] Berman, M.G., Peltier, S., Nee, D.E., Kross, E., Deldin, P.J. and Jonides, J. (2011) Depression, rumination and the default network. Social Cognitive and Affective Neuroscience, 6, 548-555. doi:10.1093/scan/nsq080

[5] Delaveau, P., Jabourian, M., Lemogne, C., Guionnet, S., Bergouignan, L. and Fossati, P. (2011) Brain effects of antidepressants in major depression: A meta-analysis of emotional processing studies. Journal of Affective Disorders, 130, 66-74. doi:10.1016/j.jad.2010.09.032

[6] Fitzgerald, P.B., Laird, A.R., Maller, J. and Daskalakis, Z.J. (2008) A meta-analytic study of changes in brain activation in depression. Human Brain Mapping, 29, 683695. doi: $10.1002 / \mathrm{hbm} .20426$

[7] Price, J.L. and Drevets, W.C. (2010) Neurocircuitry of mood disorders. Neuropsychopharmacology, 35, 192-216. doi:10.1038/npp.2009.104

[8] Raichle, M.E. (2010) Two views of brain function. Trends in Cognitive Science, 14, 180-190. doi:10.1016/j.tics.2010.01.008

[9] Lemogne, C., Mayberg, H., Bergouignan, L., Volle, E., Delaveau, P., Lehéricy, S. and Fossati, P. (2010) Selfreferential processing and the prefrontal cortex over the course of depression: A pilot study. Journal of Affective Disorders, 124, 196-201. doi:10.1016/i.jad.2009.11.003

[10] Sajonz, B., Kahnt, T., Margulies, D.S., Park, S.Q., Wittmann, A., Stoy, M., Ströhle, A., Heinz, A., Northoff, G. and Bermpohl, F. (2010) Delineating self-referential processing from episodic memory retrieval: Common and dissociable networks. Neuroimage, 50, 1606-1617. doi:10.1016/j.neuroimage.2010.01.087

[11] Kross, E., Davidson, M., Weber, J. and Ochsner, K. (2009) Coping with emotions past: The neural bases of regulating affect associated with negative autobiographical memories. Biological Psychiatry, 65, 361-366. doi:10.1016/j.biopsych.2008.10.019

[12] Kjaer, T.W., Nowak, M., Kjaer, K.W., Lou, A.R. and Lou, H.C. (2001) Precuneus-prefrontal activity during awareness of visual verbal stimuli. Consciousness and Cognition, 10, 356-365. doi:10.1006/cog.2001.0509

[13] Sheline, Y.I., Barch, D.M., Price, J.L., Rundle, M.M., Vaishnavi, S.N., Snyder, A.Z., Mintun, M.A., Wang, S., Coalson, R.S. and Raichle, M.E. (2009) The default mode network and self-referential processes in depression. Proceedings of the National Academy of Sciences of the United States of America, 106, 1942-1947. doi:10.1073/pnas.0812686106

[14] Sheline, Y.I., Price, J.L., Yan, Z. and Mintun, M.A. (2010) Resting-state functional MRI in depression unmasks increased connectivity between networks via the dorsal nexus. Proceedings of the National Academy of Sciences of the United States of America, 107, 11020-11025. doi:10.1073/pnas.1000446107

[15] Grimm, S., Boesiger, P., Beck, J., Schuepbach, D., Bermpohl, F., Walter, M., Ernst, J., Hell, D., Boeker, H. and Northoff, G. (2009) Altered negative BOLD responses in the default-mode network during emotion processing in depressed subjects. Neuropsychofarmacology, 34, 932-943. doi:10.1038/npp.2008.81

[16] Skinner, E.I. and Fernandes, M.A. (2007) Neural correlates of recollection and familiarity: A review of neuroimaging and patient data. Neuropsychologia, 45, 21632179. doi:10.1016/j.neuropsychologia.2007.03.007

[17] Svoboda, E., McKinnon, M.C. and Levine, B. (2006) The 
functional neuroanatomy of autobiographical memory: A meta-analysis. Neuropsychologia, 44, 2189-2208. doi:10.1016/i.neuropsychologia.2006.05.023

[18] Northoff, G. and Bermpohl, F. (2004) Cortical midline structures and the self. Trends in Cognitive Science, $\mathbf{8}$, 102-107. doi:10.1016/j.tics.2004.01.004

[19] Lemogne, C., Gorwood, P., Bergouignan, L., Pélissolo, A., Lehéricy, S. and Fossati, P. (2011) Negative affectivity, self referential processing and the cortical midline structures. Social Cognitive and Affective Neuroscience, $\mathbf{6}$, 426-433. doi:10.1093/scan/nsq049

[20] Cooney, R.E., Joormann, J., Eugène, F., Dennis, E.L. and Gotlib, I.H. (2010) Neural correlates of rumination in depression. Cognitive, Affective and Behavioral Neuroscience, 10, 470-478. doi:10.3758/CABN.10.4.470

[21] Järnum, H., Eskildsen, S.F., Steffensen, E.G., LundbyeChristensen, S., Simonsen, C.W., Thomsen, I.S., Fründ, E.T., Théberge, J. and Larsson, E.M. (2011) Longitudinal MRI study of cortical thickness, perfusion, and metabolite levels in major depressive disorder. Acta Psychiatrica Scandinavica, 124, 435-446. doi:10.1111/j.1600-0447.2011.01766.X

[22] Lai, C.H., Hsu, Y.Y. and Wu, Y.T. (2010) First episode drug-naïve major depressive disorder with panic disorder: Gray matter deficits in limbic and default network structures. European Neuropsychopharmacology: The Journal of the European College of Neuropsychopharmacology, 20, 676-682. doi:10.1016/i.euroneuro.2010.06.002

[23] Bai, Y., Du, L., Shen, L., Zhang, Y. and Zhang, L. (2009) GPR56 is highly expressed in neural stem cells but downregulated during differentiation. Neuroreport, 20, 918-922. doi:10.1097/WNR.0b013e32832c92d7

[24] Pizzagalli, D.A., Nitschke, J.B., Oakes, T.R., Hendrick, A.M., Horras, K.A., Larson, C.L., Abercrombie, H.C., Schaefer, J.V., Benca, R.M., Pascual-Marqui, R.D. and Davidson R.J. (2002) Brain electrical tomography in depression: The importance of symptom severity, anxiety and melancholic features. Biological Psychiatry, 52, 7385. doi:10.1016/S0006-3223(02)01313-6

[25] Bremner, J.D., Vythilingam, M., Vermetten, E. and Charney, D.S. (2007) Effects of antidepressant treatment on neural correlates of emotional and neutral declarative verbal memory in depression. Journal of Affective Disorders, 101, 99-111. doi:10.1016/j.jad.2006.10.028

[26] Fu, C.H., Williams, S.C., Cleare, A.J., Brammer, M.J., Walsh, N.D., Kim, J., Andrew, C.M., Pich, E.M., Williams, P.M., Reed, L.J., Mitterschiffthaler, M.T., Suckling, J. and Bullmore, E.T. (2004) Attenuation of the neural response to sad faces in major depression by antidepressant treatment. Archives of General Psychiatry, 61, 877-889. doi:10.1001/archpsyc.61.9.877

[27] Yildiz-Yesiloglu, A. and Ankerst, D.P. (2006) Review of $1 \mathrm{H}$ magnetic resonance spectroscopy findings in major depressive disorder: A meta-analysis. Psychiatry Research, 147, 1-25. doi:10.1016/j.pscychresns.2005.12.004

[28] Dager, S.R., Oskin, N.M., Richards, T.L. and Posse, S. (2008) Research applications of magnetic resonance spectroscopy (MRS) to investigate psychiatric disorders. Topics in Magnetic Resonance Imaging, 19, 81-96.
doi:10.1097/RMR.0b013e318181e 0be

[29] Frey, R., Metzler, D., Fischer, P., Heiden, A., Scharfetter, J., Moser, E. and Kasper, S. (1998) Myo-inositol in depressive and healthy subjects determined by frontal ${ }^{1} \mathrm{H}$-magnetic resonance spectroscopy al 1.5 tesla. Journal of Psychiatric Research, 32, 411-420. doi:10.1016/S0022-3956(98)00033-8

[30] Gruber, S., Frey, R., Mlynárik, V., Stadlbauer, A., Heiden, A., Kasper, S., Kemp, G.J. and Moser, E. (2003) Quantification of metabolic differences in the frontal brain of depressive patients and controls obtained by ${ }^{1} \mathrm{H}-\mathrm{MRS}$ at 3 tesla. Investigative Radiology, 38, 403-408. doi:10.1097/01.rli.0000073446.43445.20

[31] Coupland, N.J., Ogilvie, C.J., Hegadoren, K.M., Seres, P., Hanstock, C.C. and Allen, P.S. (2005) Decreased prefrontal myo-inositol in major depressive disorder. Biological Psychiatry, 57, 1526-1534. doi:10.1016/j.biopsych.2005.02.027

[32] Taylor, M.J., Selvaraj, S., Norbury, R., Jezzard, P. and Cowen, P.J. (2009) Normal glutamate but elevated myoinositol in anterior cingulate cortex in recovered depressed patients. Journal of Affective Disorders, 119, 186-189. doi:10.1016/j.jad.2009.02.022

[33] Kaymak, S.U., Demir, B., Oğuz, K.K., Sentürk, S. and Uluğ, B. (2009) Antidepressant effect detected on proton magnetic resonance spectroscopy in drug-naïve female patients with first-episode major depression. Psychiatry and Clinical Neurosciences, 63, 350-356. doi:10.1111/j.1440-1819.2009.01951.X

[34] Wang, Y., Jia, Y., Xu, G., Ling, X., Liu, S. and Huang, L. (2012) Frontal white matter biochemical abnormalities in first-episode, treatment-naïve patients with major depressive disorder: A proton magnetic resonance spectroscopy study. Journal of Affective Disorders, 136, 620-626. doi:10.1016/j.jad.2011.10.020

[35] Portella, M.J., de Diego-Adeliño, J., Gómez-Ansón, B., Morgan-Ferrando, R., Vives, Y., Puigdemont, D., PérezEgea, R., Ruscalleda, J., Álvarez, E. and Pérez, V. (2011) Ventromedial prefrontal spectroscopic abnormalities over the course of depression: A comparison among first episode, remitted recurrent and chronic patients. Journal of Psychiatric Research, 45, 427-34. doi:10.1016/j.jpsychires.2010.08.010

[36] Hasler, G., van der Veen, J.W., Tumonis, T., Meyers, N., Shen, J. and Drevets, W.C. (2007) Reduced prefrontal glutamate/glutamine and gamma-aminobutyric acid levels in major depression determined using proton magnetic resonance spectroscopy. Archives of General Psychiatry, 64, 193-200. doi:10.1001/archpsyc.64.2.193

[37] Horn, D.I., Yu, C., Steiner, J., Buchmann, J., Kaufmann, J., Osoba, A., Eckert, U., Zierhut, K.C., Schiltz, K., He, H., Biswal, B., Bogerts, B. and Walter, M. (2010) Glutamatergic and resting-state functional connectivity correlates of severity in major depression-The role of pregenual anterior cingulate cortex and anterior insula. Frontiers in System Neuroscience, 4, 1-10.

[38] American Psychiatric Association (1994) Diagnostic and statistical manual of mental disorders. American Psychiatric Press, Arlington. 
[39] Derogatis, L.R. (1997) SCL-90R: Administration, scoring and procedures manual for the revised version. John Hopkins University, Baltimore.

[40] Hamilton, M. (1967) Development of a rating scale for primary depressive illness. The British Journal of Social and Clinical Psychology, 6, 278-96. doi:10.1111/j.2044-8260.1967.tb00530.x

[41] Beck, A.T., Ward, C.H., Mendelson, M., Mock, J. and Erbaugh J. (1961) An inventory for measuring depression. Archives of General Psychiatry, 4, 561- 571. doi:10.1001/archpsyc.1961.01710120031004

[42] Provencher, S. (2009) LCModel and LCMgui User's Manual.

http://s-provencher.com/pub/LCModel/manual/manual.pd $\underline{\mathrm{f}}$

[43] Zhu, X., Wang, X., Xiao, J., Liao, J., Zhong, M., Wang, W. and Yao, S. (2012) Evidence of a dissociation pattern in resting-state default mode network connectivity in first-episode, treatment-naive major depression patients. Biological Psychiatry, 71, 611-617. doi:10.1016/j.biopsych.2011.10.035

[44] Davanzo, P., Yue, K., Thomas, M.A., Belin, T., Mintz, J., Venkatraman, T.N., Santoro, E., Barnett, A. and McCracken, J. (2003) Proton magnetic resonance spectroscopy of bipolar disorder versus intermittent explosive disorder in children and adolescents. American Journal of Psychiatry, 160, 1442-1452.

doi:10.1176/appi.ajp.160.8.1442 